\title{
A NEW ECONOMICAL FLUORESCENT LAMP INFORMATION TRANSMISSION SYSTEM FOR INDOOR TRACKING WITH APPLICATIONS FOR INDOOR GAMES
}

\author{
Yue Li, Adrian David Cheok \\ National University of Singapore
}

\begin{abstract}
This paper describes a light sensor-based information transmission system for indoor tracking, which uses simple fluorescent lamps, and is very cheap and economical. Fluorescent light is used as the medium to transmit information, which is encoded using a pulse-frequency modulation scheme. The user receives the data through a receiver circuit and it is passed into the wearable computer after the data is decoded.
\end{abstract}

Key words: electronic ballast, fluorescent lamp, wearable computer, wearable augmented reality

\section{INTRODUCTION}

Wearable computers and augmented reality technology are finding applications in human position guidance and tracking navigation [1]. Commonly, GPS sensors have been widely used with such interactive technologies for tracking and positioning [2], [3]. For example, GPS based tracking for wearable augmented reality has been used in the application of outdoor augmented reality computer games [4]. However, the information transmission method for wearable GPS cannot provide information indoors or in crowded urban areas [5]. One might, instead, use active badges or beacon architectures, but installing and maintaining such systems involves substantial effort and high expense [5]. Thus some other methods, which

The original version of this chapter was revised: The copyright line was incorrect. This has been corrected. The Erratum to this chapter is available at DOI: 10.1007/978-0-387-35660-0_65 
could substitute GPS sensors in a cheap and economical manner, become necessary.

Although other forms of indoor tracking such as magnetic and ultrasonic sensing are also available, they are normally for short range and highly expensive [5]. We provide another novel method by using fluorescent light based sensor, which can provide indoor tracking very cheaply with an accuracy of the same order as GPS sensors, and can be used in very wide areas. The novel system, which we describe in the paper, is inexpensive and at the same order of accuracy as wearable GPS sensors. Wearable augmented reality and interactive technologies can use this system as a valuable alternative for advance indoor tracking systems.

In this system, we consider using common and cheap fluorescent lamps [6] to transmit information for guiding and positioning, since using fluorescent lamps to provide illumination is so popular, widespread and economical. By modulating the arc frequency of the electronic ballast without any human perceptible flicker (visible spectrum is between $0.4 \mu \mathrm{m}$ to $0.7 \mu \mathrm{m}$ [7]), we can encode all the required information into the light. The user who has the developed small light receiver on her body can receive information and interact with the environment efficiently with the help of wearable augmented reality technology. The idea of using fluorescent light for transmitting information very cheaply and economically for usage in tracking and positioning with wearable augmented reality technology is first described here.

In this system, after a data stream of information is received and processed, it will be fed into the wearable computer for further processing. Also, when the wearer is at the different positions, some different graphics or virtual objects can appear at different directions. All the information will be combined and fed into the wearable computer through the serial port.

In this paper, Section 2 reviews the whole system design. Section 3 describes the application in computer games. And, a conclusion is provided in Section 4.

\section{System Design}

In this section, we will outline the system used for constructing novel and economical tracking and positioning systems using fluorescent lamps. This guiding system is divided into two parts: the transmitter and the receiver. The transmitter sends out messages encoded by the fluorescent light whose flicking is imperceptible to human vision while the receiver detects the light using a photo detector.

In the transmitter section (Figure1 (a)), information can be encoded into the light through the arc frequency variation. The frequency variation, which 
is from $35 \mathrm{kHz}$ to $40 \mathrm{kHz}$, is controlled by the microcontroller embedded in the transmitter circuit. The receiver circuit, with a photo-detector detecting the fluorescent light, processes the data which are eventually fed into the wearable computer (Figure1 (b)). With the information received, the wearable computer can tell the user what the surrounding situation is.

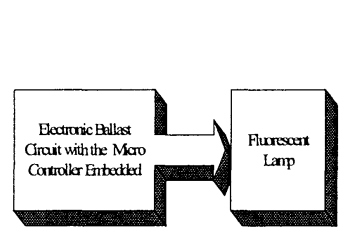

(a)

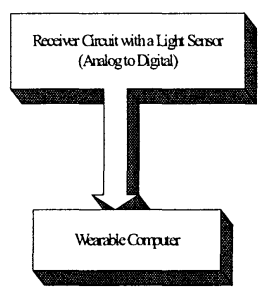

(b)

Figure1. Simple schematic scheme of the system: (a) Transmitter (b) Receiver

We will now discuss the hardware for the developed transmitter in depth (Figure 2 (a)). The lighting of the fluorescent lamp is due to arc current running through the lamp. Amplitude and frequency are the two key factors for the lamp output. Therefore, we may change the frequency of the arc current to encode all the information to the fluorescent light. If the modulation frequencies are high enough, we can transmit information without observing flickering.

The electronic ballast circuit used for the transmission purpose consists of three parts: the AC-DC rectifier, DC-AC converter and resonant filter circuit. These are detailed in Figure 3.
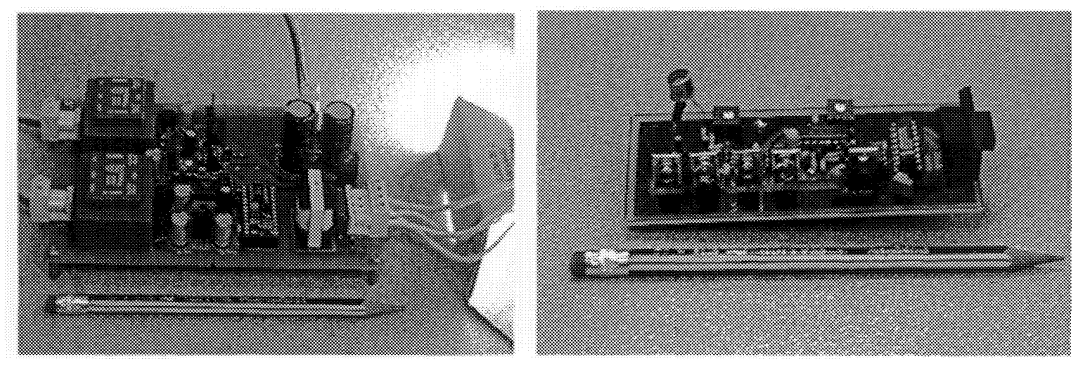

Figure 2. (a) Transmitter hardware, (b) Receiver hardware

The arc frequency of the electronic ballast is set by the two voltage controlled power MOSFET 's switching frequency. The selected frequency can be modified by switching the MOSFET IRFD110. When the MOSFET IRFD1 10 is on or off, different RC timing circuits are formed. When IRFD110 is on, the frequency is about $35 \mathrm{kHz}$; when the IRFD110 is off, the 
frequency is about $40 \mathrm{kHz}$. The two capacitors $(47 \mu \mathrm{F}, 250 \mathrm{~V}$ in Figure 3) are charged and discharged frequently so as to rectify DC to AC and light up the fluorescent lamp. However, it is not enough for us to encode the information to the light without perceptible flickering by simply switching the MOSFET IRFD110. Thus, a coding scheme is compulsory for data coding and the steady light output. We choose Manchester Coding [8] method since it is easier for use and suitable for the application here

Manchester Coding is to use two-level patterns to stand for bit " 1 " and bit "0", in which both the high-level and low-level last for one period, $\mathrm{T}_{\mathrm{sw}}$. In this system, we set $T_{\text {sw }}$ to be $1 \mathrm{~ms}$, which is the shifting period from $40 \mathrm{kHz}$ to $35 \mathrm{kHz}$. It is not difficult for almost any type of microcontroller to respond during this period. We set bit " 1 " to stand for the transition from 35 $\mathrm{kHz}$ to $40 \mathrm{kHz}$ while bit " 0 " for the transition from $40 \mathrm{kHz}$ to $35 \mathrm{kHz}$. We also set a "Start" bit which notifies the wearable computer to start processing the data.

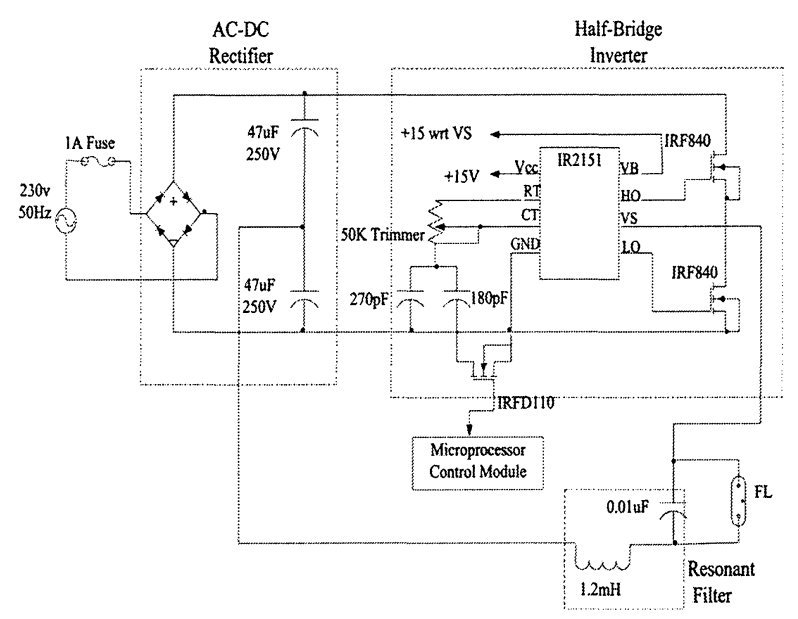

Figure 3. Electronic ballast circuit

Before the codes are fed into the gate of IRF110, all the codes from the microcontroller pass through the opto-coupler so as to separate the ground of the microcontroller and IRF110. Since the opto-coupler inverts the signals, we set the shifting from $35 \mathrm{kHz}$ to $40 \mathrm{kHz}$ as "1" bits while $40 \mathrm{kHz}$ to 35 $\mathrm{kHz}$ as " 0 " bits at this moment. Figure 4 shows a coded serial data pulse corresponding to (start bit, $0,1,1,1,0,0,0,1$ ). Note that each bit period is $2 \mathrm{~ms}\left(2 \mathrm{~T}_{\mathrm{sw}}\right)$ with a 0.5 duty cycle. The total time taken for a byte was about $(8 \times 2 \mathrm{~ms})+6 \mathrm{~ms}=22 \mathrm{~ms}$. This means that the average data rate was around 8 $/ 22 \mathrm{~ms}=363 \mathrm{bps}$. With the codes fed into the electronic ballast circuit, we can light up the fluorescent light and change the arc frequency according to the bit patterns without perceptible flickering to the human vision. 


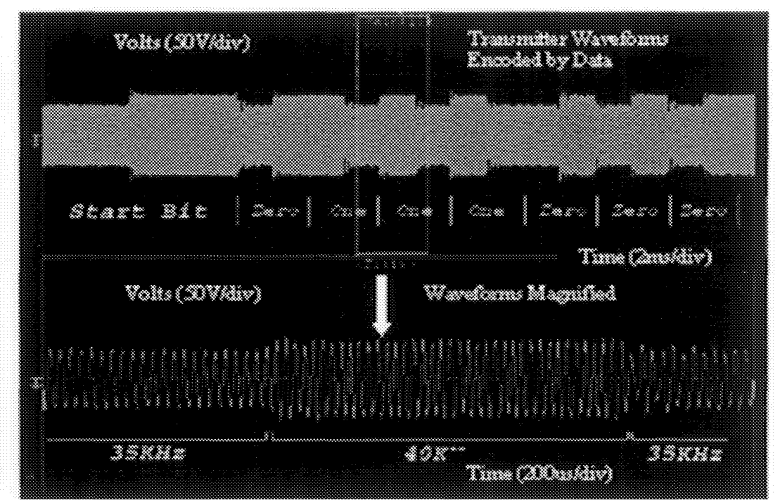

Figure 4. Manchester coded arc frequency waveforms

We will now detail how the receiver detects the fluorescent light and transforms the analog signals to the digital ones. The core part of this receiver system is the receiver circuit. Its picture is shown in the Figure 2(b) and the schematic is in Figure 5.

The light sensor, photo-detector IPL10530D, which is to provide an output voltage proportional to the light level, is used to detect the output from the fluorescent lamp in this prototype. Since the photo-detector can only sense the light intensity (when the arc output is bigger, the light intensity is higher), the received signal's frequency is doubled. While the arc frequency is from $35 \mathrm{kHz}$ to $40 \mathrm{kHz}$, the received signal's frequency is from $70 \mathrm{kHz}$ to $80 \mathrm{kHz}$.

A zero-crossing detector is built with an amplifier, which transforms the signals from the band-pass filter into square waves with the frequency (70 $\mathrm{kHz}$ to $80 \mathrm{kHz}$ ) which is unchanged from the received light frequency.

The frequencies from zero-crossing detector are tracked by a phaselocked loop IC (PLL) (NE565A) through checking the zero crossing points. As can be seen from Figure 6, through the receiver circuit, all the information received is exactly recovered to the original digital signals which were set in the program of microcontroller from the transmitter part. These signals recovered can now be fed into the wearable computer directly. Since all these waveforms were measured on the PCB directly, you may see, in microcontroller part, the noises on the signals which are caused by the power MOSFET 's switching frequency. 


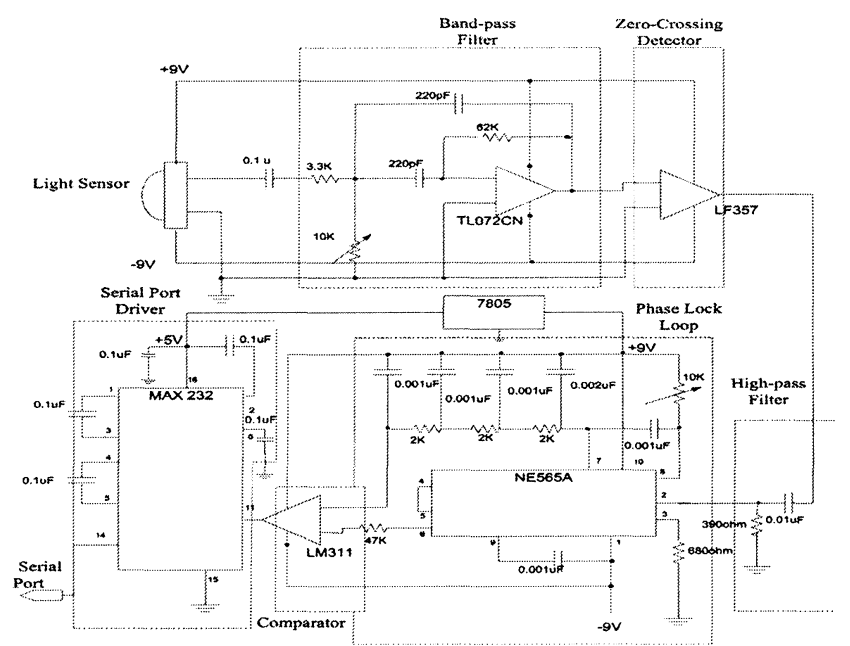

Figure 5. The schematic of the receiver circuit

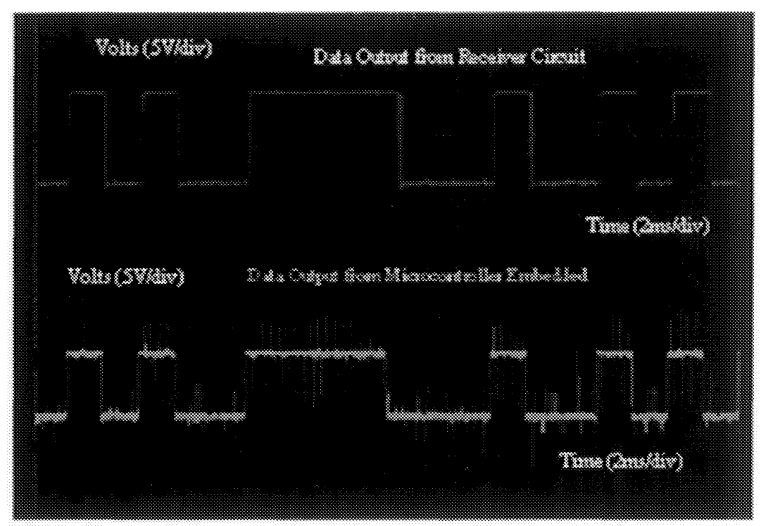

Figure 6. The data output from the receiver circuit compared to the one from microcontroller

\section{Applications in Computer Games}

In the previous section, we discussed in details of the hardware for a fluorescent light data transmission circuit. This can be used for very cheap and economical tracking for an indoor environment. With this light modulation device, the user can be positioned and tracked in the following ways. Firstly, the user can receive the information related to her position and environment. Then, she can input her destination number. The wearable computer will help the user choose the shortest the way to the destination. Note the data transmitted by the fluorescent lamps is not static, each 
fluorescent lamp can have data controlled by a base or network, which interfaces to the microcontroller of the transmission circuit.

There are many applications for this system in various fields. Its application areas can range from computer games, industrial manufacturing, urban facilities, to household usage etc. In this section, we will more focus on its applications in computer games.

With the development of the computers and other interactive technologies, new forms of games have been possible. While computer technologies have provided exciting directions for game design and game play, they have also tended to eliminate many of the social aspects of "traditional" game play [9]. With the system's help, we can change the game inside a stationary computer to a large physical space with wearable augmented reality technology. For example, imagine a maze in which several fluorescent lamps are located. The explorer who wears a wearable computer is guided to the exit of the maze by the fluorescent light. What is more, the explorer is sure to be faced with some "dangers" during her "journey" in the maze. The transmitters placed in the maze play a very important role. These transmitters are usually placed at the specific directions around the position where the messages can be received. Text messages will be displayed so as to make the explorer clearer on the complex surrounding information. For instance, at one crossing point, when the explorer receives the "Danger" and "Safe" messages at the same time, these two pieces of text messages will appear and tell the explorer at what direction the "Danger" and "Safe" really are. Therefore, the explorer will clearly know which is the "safe" path for her to take.

Moreover, a person can also act as the maze "owner" while her rivals act as the explorers. By changing the information in the microcontroller from the transmitter part, the maze owner may change friendly messages to dangerous ones or further the difficulty in the game. The "maze owner" could even suddenly change the surrounding messages to "kill" the explorer when the explorer came near to the place if the explorer did not respond to such changes quickly. All these changes could be simply done by a few revisions in the programs embedded in the transmitter circuit.

\section{Conclusions}

The work described here addresses the problem of enabling economical indoor tracking systems, similar to GPS, available. We focus on the task of the indoor navigation and positioning, where the particular aspect of the user's state that is of interest is the user's physical location. By using an 
economical light sensor to build this indoor tracking system, we are able to infer the user's location quite accurately in an indoor environment.

When data, encoded in the fluorescent light, are received by the receiver and analyzed by the wearable computer, the processed data successfully provide location and navigation messages. Specifically, this light sensor based navigation and tracking system is robust and much cheaper than those using electromagnetic, ultrasonic sensors which may be well suited to wearable computer applications.

Aside from our technical achievements of our work to date, it is significant to point out that the application of this system provides an innovative and economical form of ubiquitous games. It makes the computer games more interactive, interesting and applicable to more users due to its cheaper price and novel design. With this system, the computer games set from the desktop to incorporate the physical world brings closer the possibility of truly ubiquitous computing.

\section{Reference}

[1] S. Ertan, C. Lee, A. Wollets, H. Tan, and A. Pentland, "A Wearable Haptic Navigation Guidance System," Second International Symposium on Wearable Computers, pp. 164-165, 1998.

[2] T. Moore, "An Introduction to the Global Positioning System and its Applications," Inst. of Eng. Surveying \& Space Geodesy, Developments in the Use of Global Positioning Systems, pp. 1-6, 1994.

[3] B.Thomas, V. Demczuk, W. Piekarski,D. Hepworth, and B. Gunther, "A Wearable Computers System with Augmented Reality to Support Terrestrial Navigation," Second International Symposium on Wearable Computers, pp. 168-171,1998.

[4] B. Thomas, B.Close, J. Donoghue, J. Squires, P.D. Bondi, and W. Piekarski, "First Person Indoor/Outdoor Augmented Reality Application: ARQuake," Personal and Ubiquitous Computing. vol 6, pp. 75-86, Nov. 2002.

[5] A.R. Golding, and N. Lesh, "Indoor Navigation Using a Diverse Set of Cheap, Wearable Sensors," The Third International Symposium on Wearable Computer, pp.29-36, 1999.

[6] W. Elenbaas, Fluorescent Lamps, The Macmillan Press Ltd, 1971.

[7] J.M. Senior, Optical Fiber Communications, Principles and Practice, Prentice Hall, 1992.

[8] D.K. Jackson, T.T. Buffaloe, and S.B.Leeb, "Fiat Lux : A Fluorescent Lamp Digital Transceiver," IEEE Transactions on Industrial Applications. vol. 34 no.3 pp.625-630, May/June 1998.

[9] S. Bjork, J. Falk, R. Hansson, and P. Ljungstrand, "Pirates! Using the Physical World as a Game Board," Proceeding of Interact, pp. 1-8, 2001. 